\title{
Programmed Death Ligand 1 (PD-L1) as a Predictive Biomarker for Pembrolizumab Therapy in Patients with Advanced Non-Small-Cell Lung Cancer (NSCLC)
}

\author{
Lorena Incorvaia · Daniele Fanale - Giuseppe Badalamenti • \\ Nadia Barraco • Marco Bono - Lidia Rita Corsini • Antonio Galvano • \\ Valerio Gristina · Angela Listì · Salvatore Vieni · Stefania Gori • \\ Viviana Bazan · Antonio Russo
}

Received: March 14, 2019 / Published online: August 20, 2019

(C) The Author(s) 2019

\begin{abstract}
Recently, immunotherapy has been shown to be an effective and helpful therapeutic option for the treatment of advanced non-small-cell lung cancer (NSCLC). The activity of antitumor $\mathrm{T}$ cells may be restored through the checkpoint blockade using anti-programmed death 1 or anti-programmed death ligand 1 (PD-L1) antibodies, showing, in several cancer patients, an increased progression-free survival and overall survival compared with classical chemotherapy. As recently shown by several studies, the PD-L1
\end{abstract}

Lorena Incorvaia, Daniele Fanale and Giuseppe Badalamenti should be considered equal first authors.

Viviana Bazan and Antonio Russo should be considered equal co-last authors.

Enhanced Digital Features To view enhanced digital features for this article go to https://doi.org/10.6084/ m9.figshare.9135527.

L. Incorvaia $\cdot$ D. Fanale $\cdot$ G. Badalamenti ·

N. Barraco - M. Bono - L. R. Corsini - A. Galvano .

V. Gristina · A. Listì · A. Russo ( $\square)$

Section of Medical Oncology, Department of

Surgical, Oncological and Oral Sciences, University

of Palermo, Palermo, Italy

e-mail: antonio.russo@usa.net

S. Vieni

Division of General and Oncological Surgery,

Department of Surgical, Oncological and Oral

Sciences, University of Palermo, Palermo, Italy expression levels in tumors may offer a selection criterion for patients to predict their immunotherapy response. In particular, NSCLC patients with high tumor PD-L1 levels (proportional score $\geq 50 \%$ for first-line therapy and $\geq 1 \%$ for second-line treatment, respectively) showed better response rates to immunotherapy and longer survival in first-line therapy compared with conventional chemotherapy. PD-L1, whose expression is evaluated by using immunohistochemistry analysis, is currently the only biomarker approved for clinical use in the first- and second-line monotherapy setting and therefore plays a central role in treatment decision-making for patients with advanced NSCLC. In this review we will discuss the key role of PD-L1 as a predictive biomarker of response to pembrolizumab therapy in NSCLC patients by describing the appropriate techniques and methodologies for immunohistochemical evaluation of PD-L1 expression and

\footnotetext{
S. Gori

Department of Oncology, IRCCS Ospedale Sacro Cuore-Don Calabria, Negrar, Verona, Italy

S. Gori

Italian Association of Medical Oncology (AIOM), Bergamo, Italy

V. Bazan

Department of Biomedicine, Neuroscience and Advanced Diagnostics, University of Palermo, Palermo, Italy
} 
providing an overview of the clinical studies supporting its predictive significance.

Keywords: Checkpoint inhibitors; Lung cancer; NSCLC; PD-1; PD-L1; Pembrolizumab; Predictive biomarker

\section{INTRODUCTION}

Recently, much evidence and numerous clinical studies have shown the key role exerted by the immune system in determining an anti-tumor response in cancer patients, allowing the development of multiple therapeutic approaches aimed at stimulating and strengthening this important immune activity system [1-3]. Although further studies are currently ongoing, until now, immunotherapy has been shown to be an effective and helpful therapeutic option mainly for some tumors considered generally more immunogenic, including non-small-cell lung cancer (NSCLC), melanoma and renal cell carcinoma (RCC) [4-7]. The tumor microenvironment (TME) of these tumors is characterized by the abundant presence of several immune cells, including macrophages and $\mathrm{T}$ lymphocytes, which, if adequately activated, release cytokines, interleukins and growth factors [8]. The anti-tumor immune response induction needs three major activation signals that involve (1) the interaction between molecules with co-stimulatory function on antigen-presenting cells (APCs) and receptors present on T lymphocytes; (2) the link between tumor-associated antigens (TAAs) exposed by major histocompatibility complex (MHC) and T-cell receptor (TCR); finally, the production of inflammatory cytokines such as interleukin-12 (IL-12) and type I IFN (IFN alpha/beta) [9, 10].

The anti-tumor function exerted by the immune system through killer and proinflammatory immune cells may be repressed by the binding of some surface receptors, called immune checkpoints, with specific ligands located on cancer cells $[9,11]$. Tumor cells use this mechanism, which leads to the inactivation of tumor-infiltrating lymphocytes (TILs), to circumvent the immune surveillance and survive $[12,13]$. The activity of $\mathrm{T}$ cells may be inhibited by the interaction of the programmed death 1 (PD-1) receptor, an immune checkpoint mainly expressed on the surface of activated $\mathrm{T}$ cells, with one of its specific ligands, PD-L1 or PD-L2, expressed on tumor cells. This binding blocks the $\mathrm{T}$ cell activation signal induced by the interaction between MHC and TCR by leading to the apoptosis of $\mathrm{T}$ cells and preventing targeting of tumor cells and production of cytokines. Therefore, tumors with increased expression of the PD-1/PD-L1 axis show a poorer prognosis $[14,15]$. The activity of specific antitumor $T$ cells may be restored via the checkpoint blockade using anti-PD-1 and antiPD-L1 antibodies (Fig. 1), which determine an increase in the progression-free survival (PFS) and overall survival (OS) compared with classical chemotherapy in cancer patients [16-18]. However, to date, other immune checkpoints were identified, and some of them could be implicated in immune attacks against cancer [19]. In recent years, clinically significant progress in clinical practice resulting in improvements in prognosis of patients with advanced disease was observed thanks to the development of monoclonal antibodies specifically targeting PD-1/PD-L1. This evidence has made immunotherapy a clinically validated and effective therapeutic option for some tumors such as NSCLC, melanoma and RCC $[4,20]$.

The most important PD-1 ligand is PD-L1 (also called B7-H1), which belongs to the B7 family proteins. The tumor antigen recognition by specific T lymphocytes causes increased PDL1 expression in cancer cells via the interferon gamma (IFN- $\gamma$ ) receptor pathway, leading to tumor-adaptive resistance. Indeed, the PD-1/ PD-L1 interaction induces the cytotoxic T lymphocyte-mediated release of IFN- $\gamma$, which, binding to its specific receptor (IFNGR) present on tumor cells, causes the activation the JAK1/ 2-STAT1 signaling, resulting in an increased $P D$ $L 1$ transcription. In contrast, the inactivation of the IFN- $\gamma$ pathway makes cancer cells less vulnerable to the attack by $\mathrm{T}$ lymphocytes by reducing the response to immunotherapy $[21,22]$.

Patients with a greater likelihood of therapeutic response to antibodies blocking PD-1/ 


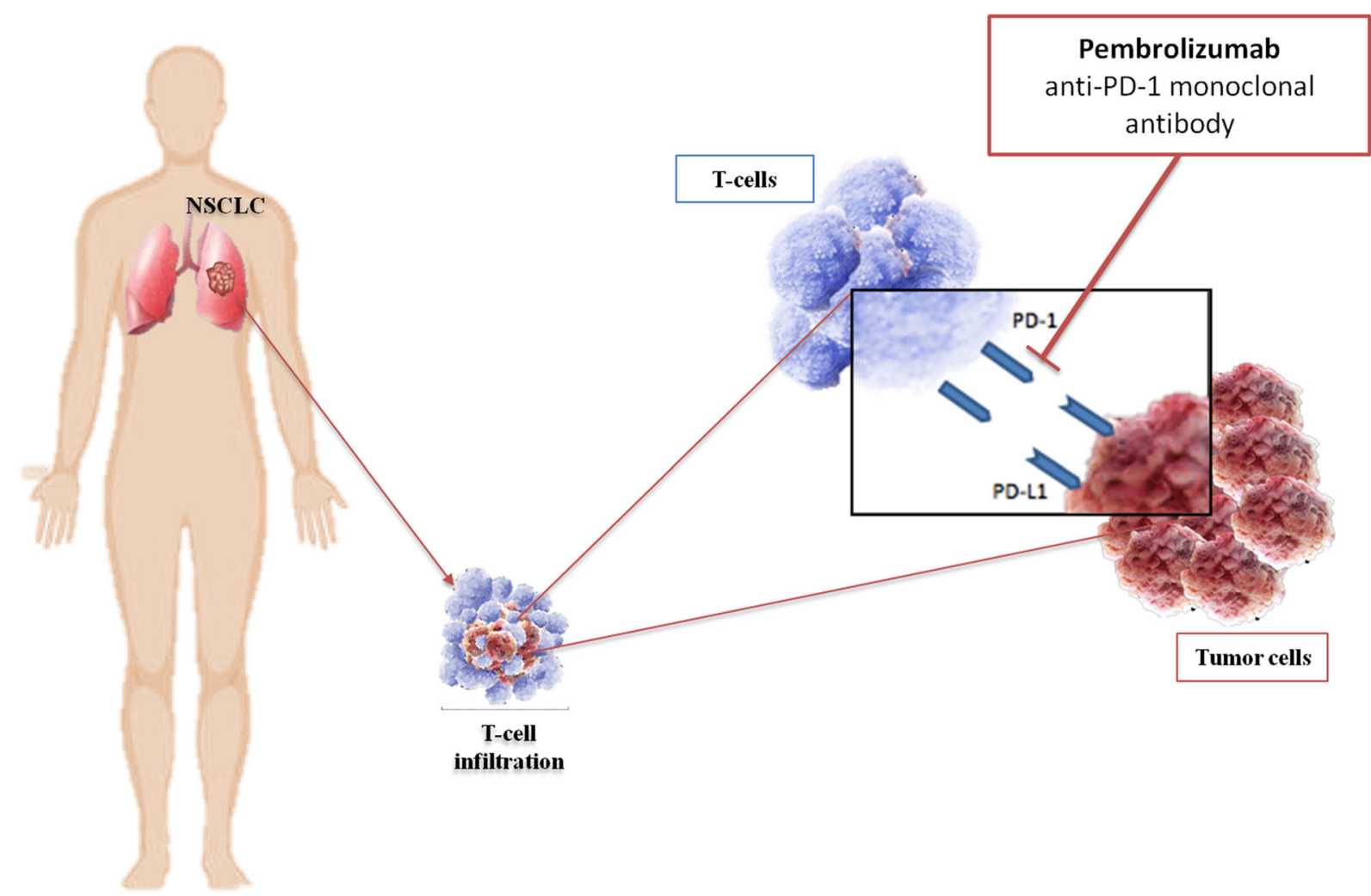

Fig. 1 PD-L1 is a transmembrane protein expressed on a variety of cell types. Binding its ligand PD-1 on activated tumor-specific CD4+ helper and CD8+T lymphocytes, PD-L1 has inhibitory functions and prevents T-cell activation. Tumor cells may overexpress PD-L1, exploiting

PD-L1 may be selected by detection of the PDL1 expression levels via immunohistochemistry (IHC) for first- and second-line monotherapy treatment decisions. Oncogenic events, such as gene amplification, or also an adaptive immune resistance mechanisms mediated by the IFN- $\gamma$ pathway may determine increased PD-L1 expression [23]. As recently shown by several studies, the PD-L1 expression levels in tumors may offer a selection criterion for patients to predict their immunotherapy response. In particular, NSCLC patients with high tumor PD-L1 levels (proportional score $\geq 50 \%$ for first-line therapy and $\geq 1 \%$ for second-line treatment, respectively) showed better response rates to immunotherapy and longer survival compared with conventional chemotherapy [24, 25]. Nivolumab (also known as Opdivo) and the inhibition of the immune system. Pembrolizumab, a monoclonal antibody blocking PD-1 through PD-1/PDL1 pathway blockade, removes the suppressive effects of PD-L1 on cytotoxic T-cells with restoration of host immunity against the tumor

pembrolizumab (also known as Keytruda) are the two most known agents blocking PD-1 currently used in cancer immunotherapy and approved by the FDA [26]. Nivolumab is a fully human anti-PD-1 antibody that was approved for the first time by the FDA in 2014 for the treatment of NSCLC, advanced melanoma and renal carcinoma. About 30\% objective response rate was observed in metastatic melanoma patients treated with nivolumab [27, 28].

Pembrolizumab is another humanized monoclonal antibody blocking PD-1 and was approved for the first time by the FDA in 2014 . The effectiveness of pembrolizumab was observed in several tumors, including NSCLC and melanoma, as shown by several studies $[29,30]$. 
Currently, assessment of PD-L1 expressions using IHC staining in formalin-fixed paraffinembedded (FFPE) tissue samples is the standard and the only one approved for clinical use. Although evaluation of PD-L1 expression by IHC has been debated, it remains the only validated biomarker. PD-L1 assessment is now fully integrated into routine clinical practice. Several detecting methods and biomarkers, such as soluble forms of sPD-1 and sPD-L1 [31], may provide new strategies in the future.

In this review, we will discuss the key role of PD-L1 as a predictive biomarker of response to pembrolizumab therapy in NSCLC patients by describing the appropriate techniques and procedures for immunohistochemical evaluation of PD-L1 expression and providing an overview of the clinical studies supporting its predictive significance.

\section{Compliance with Ethics Guidelines}

This article is based on previously conducted studies and does not contain any studies with human participants or animals performed by any of the authors.

\section{CLINICAL TRIALS SUPPORTING PD- L1 AS A PREDICTIVE BIOMARKER FOR PEMBROLIZUMAB IN PATIENTS WITH NSCLC}

\section{Evaluation of PD-L1 in Pembrolizumab NSCLC Clinical Trials}

The use of pembrolizumab for advanced NSCLC requires the identification of PD-L1 by IHC in a minimum $50 \%$ of tumor cells for the first-line setting and in $\geq 1 \%$ for second-line therapy and beyond [32].

To identify patients who could benefit from receiving immunotherapy, several PD-L1 assays have been performed. However, only the Dako/ Agilent 22c3 assay is considered as a companion diagnostic tool for pembrolizumab treatment, approved by the FDA [33].

The Dako PD-L1 IHC 22C3 pharmDx is a qualitative IHC assay based on the use of monoclonal mouse antibodies directly against PD-L1, produced by clone $22 \mathrm{C} 3$, which are able to recognize and bind the PD-L1 protein expressed on FFPE NSCLC tissues [34].

This assay requires deparaffinization, rehydration and target retrieval, which are performed by Dako PT100. Subsequently, after incubation with the monoclonal PD-L1 antibody, mouse IgG isotype control specimens were incubated with an antimouse antibody specific for the primary antibody and with a chromogen reagent that is a secondary antibody conjugated with a peroxidase. This interaction allowed us to obtain an enzymatic conversion, resulting in the precipitation of a visible reaction product at the site of antigen. The samples are counter-stained with hematoxylin, and the results are displayed with a light microscope [35].

The antibody-specific scoring used to assess the prevalence of PD-L1 positivity is the tumor proportion score (TPS) evaluated as percentage of PD-L1-positive tumor cells over total tumor cells in the sample [36]. Specifically, the positivity of PD-L1 protein expression is defined as complete or partial circumferential staining of viable tumor cell membrane [35].

The samples should be considered positive if included within a range from 1\% (low expression) to 50\% (high expression) TPS as a cutoff. Consequently, patients whose TPS was $\geq 1 \%$, if previously treated, could benefit from pembrolizumab treatment in the second-line setting, whereas the patients with $\geq 50 \%$ PD-L1positive tumor cells could be eligible for firstline treatment [34].

\section{Association Between PD-L1 TPS and Pembrolizumab Clinical Benefit in KEYNOTE-001}

The high sensitivity, repeatability and reproducibility of the PD-L1 IHC 22C3 pharmDx assay in selecting patients eligible for pembrolizumab treatment were assessed in the KEYNOTE-001 international prospective trial [37].

The KEYNOTE-001 was a phase 1 trial that evaluated the safety and activity of 
pembrolizumab in advanced NSCLC by defining and validating the PD-L1 expression levels associated with a clinical benefit of pembrolizumab treatment [37].

In this trial, 495 patients with advanced NSCLC were enrolled; 182 patients in the training group were assigned to receive three different dosages of pembrolizumab ( $2 \mathrm{mg}$ or $10 \mathrm{mg} / \mathrm{kg}$ of body weight every 3 weeks or $10 \mathrm{mg} / \mathrm{kg}$ every 2 weeks), and 313 patients were included in a validation group. IHC 22C3 pharmDx assay was used to determine the PDL1 expression evaluated as the percentage of neoplastic cells with staining for membranous PD-L1. PD-L1 positivity was defined as membranous staining in $\geq 1 \%$ of cells. The results showed that a TPS $\geq 50 \%$ was associated with a higher response rate and a median PFS and OS in both previously treated and untreated patients compared with a TPS value $<50 \%$, indicating that in these patients immunotherapy with pembrolizumab could be a successful therapeutic option [37].

\section{Patient Selection Based on PD-L1 Expression as an Eligibility Criterion in Phase 3 Pembrolizumab Monotherapy Trials (KEYNOTE-010; KEYNOTE-024; KEYNOTE-042)}

KEYNOTE-010 was a multicenter trial that randomized patients to receive pembrolizumab at two dosing schedules versus docetaxel, 1034 previously treated advanced NSCLC patients having $\geq 1 \%$ of tumor cells staining positive for PD-L1 expression (TPS 1\%) who had progressed after at least two cycles of platinum-based chemotherapy [38]. Patients were randomized in a 1:1:1 ratio to receive pembrolizumab at $2 \mathrm{mg} / \mathrm{kg}$, pembrolizumab at $10 \mathrm{mg} / \mathrm{kg}$ or docetaxel at $75 \mathrm{mg} / \mathrm{m}^{2}$ every 3 weeks [38].

The PD-L1 expression levels were detected by Dako PD-L1 IHC 22C3 assay, and patients were stratified by PD-L1 status into weak (TPS range $1-49 \%$ ) and strong (TPS $>50 \%$ or more). The primary objectives were to compare the OS and PFS in the strongly and weakly PD-L1-positive patients treated with pembrolizumab compared with docetaxel. In the overall population, the results showed a statistically significant OS improvement in patients who received pembrolizumab $2 \mathrm{mg} / \mathrm{kg}$ versus docetaxel [(HR) $0.71,95 \%$ CI $0.58-0.88 ; p=0.0008$ ] or pembrolizumab $10 \mathrm{mg} / \mathrm{kg}$ compared with docetaxel $(0.61,0.49-0.75 ; p<0.0001)$. In patients with PD-L1 expression of $\geq 50 \%$, OS was significantly longer with pembrolizumab versus docetaxel, median 14.9 months vs. 8.2 months, respectively (HR 0.54, 95\% CI 0.38-0.77; $p=0.0002$ ), with pembrolizumab $2 \mathrm{mg} / \mathrm{kg}$ and 17.3 months vs. 8.2 months $(0.50,0.36-0.70 ; p<0.0001)$ with pembrolizumab $10 \mathrm{mg} / \mathrm{kg}$. This result suggested pembrolizumab as a valid treatment option for second-line treatment in these patients with advanced NSCLC appropriately selected on the basis of the PD-L1 value [38].

Recently, based on the Keynote-010 results, an exploratory analysis compared outcomes in patients whose PD-L1 expression was performed starting from archived tumor samples compared with newly collected tumor specimens. Four hundred fifty-five (44\%) out of 1033 analyzed patients were enrolled based on archival samples and $578(56 \%)$ on newly collected tumor samples. Approximately $40 \%$ of archival samples and $45 \%$ of newly collected tumor samples were PD-L1 TPS $\geq 50 \%$.

At a median follow-up of 31 months, the OS HRs for archival versus newly collected tumor samples were $0.64(95 \%$ CI $0.45,0.91)$ and 0.40 (95\% CI $0.28,0.56)$ in patients with PD-L1 TPS $\geq 50 \%$ and, among those with PD-L1 TPS $\geq 1 \%$, were $0.74(95 \%$ CI $0.59,0.93)$ and 0.59 (95\% CI $0.48,0.73)$. These results showed that pembrolizumab compared with docetaxel improves OS in both newly collected and archival tumor samples from NSCLC patients [39].

KEYNOTE-024 was an open-label phase 3 trial, where PD-L1 expression on $\geq 50 \%$ of tumor cells represented an eligibility criterion to enroll previously untreated advanced NSCLC patients (EGFR/ALK wild type) to receive pembrolizumab (at a fixed dose of $200 \mathrm{mg}$ every 3 weeks) or a platinum-based doublet at the investigator's choice [24].

This study was carried out on 305 patients who were randomized to receive treatment with pembrolizumab for 35 cycles or 4-6 cycles at the investigator's choice of platinum-based 
chemotherapy. Fifty percent or greater TPS values were detected in $30.2 \%$ of examined patients. The median PFS was significantly longer in the pembrolizumab group, 10.3 months [95\% confidence interval (CI) 6.7-not reached] compared with the chemotherapy group, 6.0 months (95\% CI 4.2-6.2) (HR 0.50; 95\% CI 0.37-0.68; $p<0.001)$. The estimated rate of OS was significantly longer in the pembrolizumab than in the chemotherapy group (HR 0.60; 95\% CI $0.41-0.89 ; p=0.005), 80.2 \%$ versus $72.4 \%$, respectively. Based on these results, pembrolizumab was approved by the FDA for firstline treatment in patients with advanced NSCLC and $\geq 50 \%$ PD-L1 expression without EGFR mutations or ALK rearrangements [24, 40].

An update of the OS analysis of the KEYNOTE-024 study was recently performed. At data cutoff (July 10, 2017; median follow-up, 25.2 months), the median OS was 30.0 months (95\% CI 18.3 months to not reached) with pembrolizumab and 14.2 months (95\% CI 9.8-19.0 months) with chemotherapy (HR 0.63; 95\% CI 0.47-0.86), indicating that first-line pembrolizumab monotherapy continues to give an OS benefit over chemotherapy in patients with advanced NSCLC without EGFR/ALK aberrations [41].

Several ongoing clinical trials are being evaluated to assess pembrolizumab monotherapy's role as frontline treatment in patients with NSCLC. Among these trials, KEYNOTE-042 is the largest clinical trial; it was presented by Lopes et al. on April 2018 in ASCO's Plenary Session. Through the evaluation of monotherapy with pembrolizumab versus chemotherapy as the regimen in treatment-naive patients with NSCLC with PD-L1 $\geq 1 \%$, this study has already achieved its primary end point, represented by a statistically significant advantage in terms of OS [40].

This open-label phase III study included 1274 patients with PD-L1 TPS $\geq 1 \%$ who were randomly assigned to receive pembrolizumab $200 \mathrm{mg}$ every 3 weeks for up to 35 cycles or platinum-based chemotherapy (carboplatin AUC of $5-6$ plus paclitaxel at $200 \mathrm{mg} / \mathrm{m}^{2}$ or pemetrexed $500 \mathrm{mg} / \mathrm{m}^{2}$ ) every 3 weeks for $4-6$ cycles.
Primary end points were OS in patients with TPS $\geq 50 \%, \geq 20 \%$ and $\geq 1 \%$. At a median follow-up of 12.8 months, the median OS in the pembrolizumab versus chemotherapy arm was 20.0 months vs. 12.2 months among patients with PD-L1 TPS $\geq 50 \%$ (HR 0.69; $P=0.0003$ ), 17.7 months vs. 13.0 months among those with a TPS $\geq 20 \%$ (HR $0.77 ; p=0.0020$ ) and 16.7 months vs. 12.1 months among those with a TPS $\geq 1 \%$ (HR 0.81, $P=0.0018$ ).

Based on these results, on April 2019, the indication of pembrolizumab was extended to patients with advanced NSCLC without EGFR/ ALK aberrations and with low PD-L1 TPS [42].

\section{PD-L1 Expression in Studies Evaluating Pembrolizumab Plus Chemotherapy (KEYNOTE-021 cohort G; KEYNOTE-189; KEYNOTE-407)}

Although the PD-L1 biomarker has been associated with better hazard ratios for benefit with increasing expression levels, several challenges remain regarding the use of $\mathrm{PD}-\mathrm{L} 1$ as a predictive biomarker, especially in recent studies evaluating the combination of immune checkpoint inhibitors (ICIs) with chemotherapy as effective first-line treatment in advanced NSCLC. To date, the majority of trials have established that the higher the level of tumor PD-L1 expression indicating the presence of an antitumor immune response, the more likely it will be that the patient will benefit from treatment with pembrolizumab [43]. However, the role of this antitumor immune response might not be such an important factor for survival outcomes in patients treated with chemotherapy, since chemotherapy seemed to induce immunosuppression by inhibiting the antitumor immune response [44].

As a matter of fact, a Danish retrospective study evaluating 204 treatment-naive advanced NSCLC patients who were treated with platinumbased chemotherapy showed no significant association between PD-L1 expression and survival in patients receiving platinum-based chemotherapy, in contrast to the immunotherapy agents [44]. Thus, considering that some patients respond despite low PD-L1 expression 
[45] and the lack of a standardized method for evaluating this expression [46], the apparent significant, albeit not unequivocal, relationship between PD-L1 expression and responsiveness to ICIs should be accepted with some caution.

The updated efficacy findings from cohort $G$ of the multicohort phase 1/2 KEYNOTE-021 study, which evaluated the association of pembrolizumab with pemetrexed-carboplatin vs. chemotherapy alone as a safe and effective firstline therapy for advanced non-squamous NSCLC [47], have recently confirmed the noteworthy survival benefit regardless of tumor PDL1 expression observed with the addition of pembrolizumab to chemotherapy regimens in the phase 3 KEYNOTE-189 study [48]. The phase I portion of Keynote- $021 \mathrm{G}$, one of the first trials exploring the toxicity and clinical activity of combination chemotherapy and immunotherapy, paved the way for the KEYNOTE-021G study, which represented the first randomized phase-2 trial in advanced treatment-naïve nonsquamous NSCLC to assess and to demonstrate the benefit of adding immunotherapy to standard of care chemotherapy, stratifying by PD-L1 TPS $(\leq 1 \%$ or $\geq 1 \%)$. The primary end point (overall response rates, ORR) along with all the secondary end points (PFS, duration of response, OS and correlation between PD-L1 expression levels and antitumor activity) have proved to be significantly higher in those who received pembrolizumab with chemotherapy rather than chemotherapy alone. Even those with PD-L1 expression $<1 \%$ appeared to derive benefit from the combination strategy.

Consequently, a larger patient population adequately powered for survival end points was included in the awaited confirmatory doubleblind phase 3 KEYNOTE-189 study, which compared the combination of pembrolizumab or placebo plus pemetrexed and a platinumbased drug in patients with advanced nonsquamous NSCLC with any level of PD-L1 level expression, demonstrating for the first time a significant OS benefit with the addition of immunotherapy to chemotherapy in the firstline setting. Exploratory end points included the effect of PD-L1 expression on efficacy and patient-reported outcomes. The response rate and the OS benefit were higher across all categories of PD-L1 patients receiving the combination of chemotherapy with pembrolizumab. Despite the higher response rate in patients with PD-L1 TPS of $\geq 50 \%$ and a clear PFS benefit shown among the PD-L1 TPS $\leq 1 \%$, baseline positive PD-L1 expression levels were not mandatory for treatment with a reported PD-L1 TPS $\geq 1 \%$ in $63.0 \%$ of the patients. Interestingly, in a subset analysis based on PDL1 expression, survival benefit with chemotherapy and pembrolizumab was greater in patients with tumor PD-L1 TPS $\geq 50 \%$ (HR 0.42 ) than in patients with lower PD-L1 TPS (HR 0.55) or no PD-L1 expression (HR 0.59). Even though the relatively greatest benefit described in the subgroup with a PD-L1 TPS $\geq 50 \%$ appears to be greater than the survival benefit with pembrolizumab alone compared with chemotherapy (HR 0.60 in KEYNOTE 024 and HR 0.69 in KEYNOTE 042, respectively) and the hazard ratio for PFS crossed the boundary for those with a PD-L1 TPS $\leq 1 \%$, the significant survival benefit in favor of the pembrolizumab combination was associated with similar hazard ratios across all PD-L1 subgroups, including PDL1 negative patients, in the KEYNOTE-189 trial.

Likewise, the data from the double-blind phase 3 KEYNOTE-407 trial [49], which tested the addition of pembrolizumab to standard chemotherapy with carboplatin and either paclitaxel or nab-paclitaxel in patients with previously untreated metastatic squamous NSCLC, seemed to complement those of the non-squamous KEYNOTE-189 trial showing a relationship between greater PD-L1 expression and longer PFS along with a reduction in the rate of disease progression more proportionally linked to PD-L1 expression levels. The improvement of all efficacy measures statistically emerged across all PD-L1 expressing subgroups $(<1 \%, 1-49 \%$ and $\geq 50 \%)$ with the greatest benefits for high-expression PD-L1 patients, although the OS upper limit of the 95\% confidence interval did cross the boundary for the subgroup with a TPS of $\geq 50 \%$. Remarkably, when indirectly comparing the KEYNOTE-407 trial to first-line pembrolizumab alone phase-3 trials, the survival benefit of the combination of pembrolizumab with chemotherapy with tumor PD-L1 expression 
$\geq 50 \%$ (HR 0.64) did not appear to be better than pembrolizumab alone, suggesting that an added survival benefit in favor of the combination strategy may not be necessarily observed in squamous cell patients.

Nevertheless, the magnitude of survival benefit was similar across all PD-L1 subgroups, leading to the fast approval of combination therapy with pembrolizumab regardless of PDL1 status. Hence, the predictive role of PD-L1 status appeared to fail in both trials of immunechemotherapy combinations, and its clinical usefulness may be even less clear in the combination strategy. Indirect across-trial comparisons should be considered with a high level of caution and whether the combination treatment is better than monotherapy in patients with tumors with PD-L1 expression of $\geq 50 \%$ remains unknown. Therefore, prospective studies specifically assessing the role of combination strategy and directly comparing the use of chemotherapy in addition to pembrolizumab with pembrolizumab alone in patients with tumor PD-L1 expression $\geq 50 \%$ are warranted to definitely answer this question.

\section{ANALYTICAL CONSIDERATIONS}

Recently, the encouraging activity of different immunotherapeutic agents approved for the treatment of advanced NSCLC patients led the research laboratories to develop appropriate assays able to detect the immune checkpoint targets on tumor specimens. Different targeted assays were developed in association with the specific immunotherapeutic agents to reduce diagnostic errors and improve cost-effective test platforms [50].

The first IHC assays approved by the US Food and Drug Administration (FDA) aimed to detect the PD-L1 protein present in the histologic sections or cytology specimens with the final aim of selecting those patients who were best candidates for immune checkpoint inhibitors [51]. This laboratory approach is based on the ability to recognize the variable domain of the primary antibody and bind the specific epitope. The subsequent link with a second antibody will trigger a chemical reaction generating a signal enhancement. Certainly, we cannot define IHC as a recent technique; however, IHC staining allows easily identifying different cell membrane, cytoplasmic or nucleus areas as compared with fluorescence in situ hybridizations (FISH). With only the use of a microscope, IHC identifies the expression of proteins on tumor cells from different preparations, including FFPE tissue blocks, fluids and cytologic aspirates [52]. Among the different used antibodies, the 5H1, 7G11, 015, 22C3 and 28-8 antibodies bind to the extracellular domain, whereas SP142, SP263, E1L3N and 9A11 bind to the intracellular domain. Some of these antibodies have been standardized and validated for PD-L1 expression testing, while others, such as E1L3N, are most commonly used in laboratory developed tests (LDT).

In the setting of advanced NSCLC, the IHC evaluation of PD-L1 expression represents a predictive biomarker of response to PD1/PD-L1 checkpoint inhibitors, including pembrolizumab [53]. In particular, in 2015, the FDA, the European Medicines Agency (EMA) and the Japanese Ministry of Health, Labour and Welfare (MHLW) recommended PD-L1 22C3 pharmDx (Dako, Glostrup, Denmark) as a companion diagnostic test associated with pembrolizumab [54]. Among PD-L1 IHC tests, beyond the 22C3 assay associated with pembrolizumab, four other companion or complementary diagnostic assays have been developed and used to determine PD-L1 expression in NSCLC. These include PD-L1 28-8, SP142, SP263 and 73-10, associated with nivolumab, atezolizumab, durvalumab and avelumab, respectively, overall differing from the used primary monoclonal antibody, destined platform, detection system and scoring methods with different thresholds of positivity validated in clinical trials.

The PD-L1 28-8 pharmDx assay (Agilent technologies/Dako) uses clone 28-8, a rabbit IgG4, as a human anti-PD-L1 monoclonal antibody. This assay allows detecting the expression of PD-L1 in case of treatment with nivolumab and is considered a complementary diagnostic test. Nivolumab, approved by FDA and EMA for advanced NSCLC patients, is a human G4 immunoglobulin targeting PD-1 receptor, thus 
Table 1 IHC PD-L1 assay comparison

\begin{tabular}{|c|c|c|c|c|c|}
\hline $\begin{array}{l}\text { Assay Antibodies } \\
\text { PD-L1 clone }\end{array}$ & $\begin{array}{l}\text { Staining } \\
\text { platform }\end{array}$ & FDA designed & $\begin{array}{l}\text { Immunotherapy } \\
\text { drug }\end{array}$ & $\begin{array}{l}\text { PD-L1 } \\
\text { binding } \\
\text { domain }\end{array}$ & $\begin{array}{l}\text { Clinical cutoff(s) for } \\
\text { PD-L1 expression }\end{array}$ \\
\hline $28-8$ & Dako Link 48 & $\begin{array}{l}\text { Complementary } \\
\text { diagnostic }\end{array}$ & Nivolumab & Tumor cells & $\geq 1 \%, \geq 5 \%$ \\
\hline $22 \mathrm{C} 3$ & Dako Link 48 & Companion & Pembrolizumab & Tumor cells & $\geq 1 \%, \geq 50 \%$ \\
\hline SP142 & $\begin{array}{l}\text { Ventana } \\
\text { Benchmark or } \\
\text { Ultra }\end{array}$ & $\begin{array}{l}\text { Complementary } \\
\text { diagnostic }\end{array}$ & Atezolizumab & $\begin{array}{l}\text { Tumor cells } \\
\text { and/or } \\
\text { Infiltrating } \\
\text { immune } \\
\text { cells }\end{array}$ & $\begin{array}{l}\geq 1 \%, \geq 5 \%, \geq 50 \% \\
\geq 1 \%, \geq 5 \%, \geq 10 \%\end{array}$ \\
\hline SP263 & $\begin{array}{l}\text { Ventana } \\
\text { Benchmark or } \\
\text { Ultra }\end{array}$ & No designed & Durvalumab & Tumor cells & $\geq 25 \%$ \\
\hline $73-10$ & Dako Link 48 & $\begin{array}{l}\text { Under } \\
\text { development }\end{array}$ & Avelumab & Tumor cells & $\geq 1 \%, \geq 5 \%, \geq 80 \%$ \\
\hline
\end{tabular}

Clinical cutoff(s) for PD-L1 expression of five-assay comparison

blocking the binding with its natural ligands, PD-L1 and PD-L2, to stimulate the anti-tumor immune response. PD-L1 28-8 pharmDx assay is an FDA-approved and European Conformity (CE) in vitro device (IVD) that is performed from the Autostainer Link 48 platform (Agilent technologies/Dako).

The Ventana SP142 PD-L1 assay was built with a rabbit monoclonal antibody to recognize the intracellular domain of PD-L1 protein. It has been approved by the FDA for the treatment of some tumors with atezolizumab. The SP142 is only approved for the detection and amplification assay related to Ventana Medical System Inc.

PD-L1 SP263 uses SP263, a rabbit primary monoclonal antibody, to detect PD-L1 expression in membranous and/or cytoplasmatic regions of tumor cells subjected to durvalumab therapy. Durvalumab is a human G1 kappa immunoglobulin that interacts with PD-L1 to avoid the link with PD-1 and CD80. This assay was generated from a close collaboration of Ventana Medical System Inc., Roche and AstraZeneca. PD-L1 73-10 Assay, developed by Dako (Agilent Technologies), has employed a rabbit recombined 73-10 clone and is associated to the use of avelumab (Table 1).

Each commercially validated assay is designed for use on a particular staining platform $[55,56]$.

The performance of the different PD-L1 IHC assays was tested during the Blueprint project to compare the analytical validity and diagnostic ability to detect PD-L1 protein expression on tumor samples of advanced NSCLC patients [57].

Thirty-nine NSCLC FFPE tissue samples were analyzed, 18 provided by Dako sectioned at $4 \mu \mathrm{m}$ and 21 by Ventana at $10 \mu \mathrm{m}$, most of them derived from surgical specimens, but a few from biopsies. Every diagnostic assay produced a staining on the basis of the PD-L1 expression on tumor cell (TC) membranes, and only the PD-L1 SP 142 included a measure of infiltrating PD-L1positive immune cells (ICs) in addition to TCs. These parameters were estimated to calculate the TPS for each tumor slide. For the first time, the BLUEPRINT project simultaneously investigated both analytical and clinical diagnostic aspects by comparing staining cutoffs of each assay with the associated treatment. The raw 
percentage of tumor and immune cells stained by each assay was determined, and cutoffs for each assay, as recommended by the manufacturers upon FDA approval, were used as follows: $>/<1 \%$ of tumor cell staining for the $28-8$ and $22 \mathrm{C} 3$ assays; $>/<25 \%$ of staining for the SP263 assay; tumor- and immune-cell scoring of TCO-3/ICO-3 for the SP142 assay, and tumorand immune-cell scoring of TC1/IC1 for the SP142 assay.

From these comparative studies, we know that the PD-L1 28-8, 22C3 and SP263 tests provided almost the same diagnostic performance, while the PD-L1 SP142 assay provided less evidence in the tumor cell staining. Furthermore, this comparative analytic study showed poor concordance regarding tumor-infiltrating ICs [57]. On the basis of these preliminary data, a larger, more comprehensive phase 2 study was designed. This trial also included the PD-L1 73-10 assay, tested 81 NSCLC tumor samples and expanded the number of pathologists involved in the PD-L1 detection (25 versus 3 pathologists). The images were studied using digital microscopy accessible from a Web system based on standard criteria and obtained results showing good concordance among the different pathologists.

The result of a phase II trial confirmed the similar performance obtained by PD-L1 28-8, 22C3 and SP263 in detecting PD-L1 expression on tumor cells and confirmed that the SP142 assay has a reduced ability to stain tumor cells. The PD-L1 73-10 assay was compared for the first time with the other four assays included in the phase II of BLUPRINT project, showing an increased staining of tumor cells. Thus, the possibility to choose among these tests could not be based on the diagnostic performance, but only on the associated immunotherapeutic agent used for clinical treatment [58].

Regarding the procedures related to the PDL1 22C3 pharmDx assay, these are standardized by the Dako Automated Link 48 platform. Obviously, the first steps are represented by adequate preparations of tumor slides, and the gold standard is the FFPE tissue.

A serious problem in the development of the diagnostic assays for the PD-L1 evaluation is the exclusion of cytologic samples from clinical trials. In fact, PD-L1 assays are currently only approved for use for histologic samples, although most NSCLC patients are diagnosed by cytologic specimens that require a less invasive approach, such as computed tomographyguided fine-needle aspiration and endobronchial ultrasound (EBUS)-guided aspiration biopsies [59].

Different studies compared the evaluation of PD-L1 through IHC on different sample types, such as tumor resection, biopsy and cytologic samples, to define which is the most suitable material to evaluate the expression of PDL1.

Skov et al. analyzed 87 paired samples, including histologic and cytologic samples from the same lesion by means of two IHC assays, 28-8 and 22C3 by Dako. Obtained results showed an elevated concordance between two types of materials, ascribing the slight discrepancy to the staining heterogeneity of PD-L1 on histologic specimens. Therefore, evaluation of the PD-L1 expression scoring would be feasible on both cytologic and histologic tumor specimens using the Dako assays (28-8 and 22C3) [60].

Heymann et al. [61] compared PD-L1 expression in cytology, small biopsy and surgical resection of NSCLC specimens with 22C3 by Dako. Two hundred fourteen samples, including surgical resection, biopsy specimens (derived from computed tomography-guided core needle biopsy, endobronchial forceps biopsy or histologic biopsy) and cytology specimens (derived from EBUS-FNA, FNA, thoracentesis or pericardiocentesis), were collected from 188 patients. No difference was revealed between the PD-L1 expression on small biopsy and surgical resection, while PD-L1 expression was higher in cytologic than histologic materials. Results showed that the evaluation of PD-L1 on cytologic specimens is important in order not to exclude from clinical treatment those patients that normally are not accepted because their tumor tissue is not available [61].

The study performed by Wang et al. [62] analyzed a total of 1419 specimens with diagnosis of NSCLC, distributed in 371 cytology cell blocks, 809 small biopsies and 239 surgical resections. Cytologic samples were divided into 
endo-bronchial ultrasound-guided transbronchial needle aspiration (EBUS TBNA), endoscopic ultrasound-guided fine-needle aspiration (EUS-FNA), fine-needle aspiration, pleural/pericardial fluid and broncho-alveolar lavage. In cytologic specimens, the expression of PD-L1 with 50\% TPS was higher than in surgical specimens or weakly higher than in small biopsy. The comparison between surgical samples or biopsies and cytologic samples was more difficult to interpret because in both EBUS/EUS/FNA specimens and biopsy samples cancer cells were very cohesive, whereas cancer cells in fluid cytology were difficult to distinguish from macrophages and mesothelial cells. These problems can be solved by comparing with hematoxylin/eosin (H\&E)-stained slides and through evaluation of nuclear properties to make the interpretation of PD-L1 staining easier. Therefore, it was possible to demonstrate that cytology specimens were appropriate for PD-L1 staining by IHC [62].

Despite the obtained excellent results, however, a standardization is necessary for the evaluation of PD-L1 by IHC on cytologic specimens due to different methods used for cell collection and fixation.

Another hot topic is represented by the comparison of archival versus fresh biopsy for the detection of PD-L1 expression by IHC. In the ATLANTIC trial, Midha et al. [63] evaluated PD-L1 expression and compared 112 archival tumor samples ( $<3$ years) with recently acquired tumor samples ( $<3$ months). This study showed a high concordance (76.2\%) between archival and fresh tumor samples. A reanalysis of the KEYNOTE-010 trial allowed evaluating the PD-L1 expression levels in 578 re-biopsy samples by comparing them with 456 previously analyzed archival samples. The rate of PD-L1 levels $\geq 50 \%$ was overlapped in archival and re-biopsy samples. These data, which obviously must be increased, could suggest that re-biopsy may not be necessary for the IHC evaluation of PD-L1 when it is possible to have archival samples $\leq 3$ years old and 6 months of staining [59].

The protocol started with the use of the Dako Envision Target retrieval solution, used for deparaffinization, rehydration and epitope retrieval on formalin-fixed samples. The working solution must be incubated in Dako PT link at $97^{\circ} \mathrm{C}$ for a time ranging from 20 to $40 \mathrm{~min}$ and successively at $65^{\circ} \mathrm{C}$ to cool. The successive steps involve the use of buffers to wash the slides before staining. At this point, the slides could be placed on a Dako Autostainer Platform to proceed with the staining phase. According to the immunohistochemical staining procedure, the slides are counterstained with hematoxylin, and the primary antibody detection is performed using a light microscope (https:// www.agilent.com/cs/library/packageinsert/pub lic/115030003.PDF).

Counterstaining with hematoxylin and eosin is indispensable to verify the stain quality and preservation of the analyzed tumor specimens before assessing the comparison with both positive and negative controls [64].

It is essential to remember that, although this assay is standardized and validated, many serious errors occur [65]. For this reason, it is recommended that each laboratory should include several controls (https://www.agilent. com/cs/library/usermanuals/public/29158_pd11-ihc-22C3-pharmdx-nsclc-interpretation-man ual.pdf). During the preparation of the tissue samples, tumor slides containing two cell lines, considered as positive and negative controls, must be entered. One of these (positive control) must moderately express PD-L1 (e.g., NC$\mathrm{H} 266$ ); the other acting as a negative control must be negative for PD-L1 expression (e.g., MCF-7). In addition, every laboratory should also have FFPE cell blocks of human tonsils or placenta as an external comparator for the positive control. Indeed, FFPE human tonsils or placenta cell blocks are an ideal area for PD-L1 analysis, since this immune checkpoint is expressed on dendritic cells, macrophages, mast cells, $\mathrm{T}$ and $\mathrm{B}$ lymphocytes, endothelial and tumor cells [66, 67]. An additional positive control is considered good laboratory practice if it is processed in the same manner as the samples. Indeed, a comparison between cell lines showing high PD-L1 expression and human tissues allows detecting a calibrated sample for tissue staining, avoiding mistakes related to possible minor staining associated with the cell line. Furthermore, a negative control is crucial 
to ascertaining that any negative staining is related to the effective absence of PD-L1 expression on tumor samples.

The PD-L1 expression value was measured by the TPS scoring method [68].

The percentage expression of TPS allows quantifying the partial or complete membrane staining of viable tumor cells in the context of all viable tumor cells, both positive and negative, present in the advanced NSCLC sample. For each specimen, on the PD-L1-stained slide, indistinct tumor biopsy or resection tissue, as long as there is adequate biologic material, tumor cells must show complete or not complete membrane staining ( $\geq 1+$ intensity) with a minimum of 100 viable cells. Although staining on the cytology specimens can also be done, none of the trials using clone 22C3 have currently validated these tests.

The scoring is interpreted as follows: TPS $<1 \%$ corresponds to lack of PD-L1 expression; TPS between 1 and 49\% indicates the presence of PD-L1 expression, while TPS $\geq 50 \%$ is associated with high PD-L1 expression [37]. This score method excludes stained tumor-associated immune cells, normal/non-neoplastic cells and necrotic cells.

In patients with NSCLC, the value of the PDL1 IHC 22C3 pharmDx assay in predicting treatment response to pembrolizumab has been largely demonstrated [38, 69]. In general, increased PD-L1 expression (higher TPS) was associated with a higher ORR and PFS with favorable outcome. The presence of immune cells, including macrophages and lymphocytes, may generate difficulties in the data interpretation during IHC staining; therefore, these cells should be excluded from the scoring in NSCLC. However, since PD-L1 plays a critical role as a predictive biomarker, its expression must inevitably include that observed on immune cells. Indeed, the poor data reproducibility for the evaluation of the mononuclear immune cell density score (MIDS) and ratio of PD-L1-expressing immune cells compared with that of all tumor cells have been bypassed thanks to the combined positive score (CPS). The CPS reproducible method, resulting from the combination of TPS and MIDS, allowed evaluating the number of all PD-L1-expressing cells represented by tumor cells, lymphocytes and macrophages inside all tumor cells. The CPS is the percentage ratio of all PD-L1-stained tumor cells, lymphocytes and macrophages compared with that of all tumor cells.

Kulangara et al. [70] studied the possibility of introducing CPS as an alternative scoring method across four tumor types (gastric cancer, urothelial tumor, triple-negative breast and ovarian cancer), involving many pathologists to evaluate the reliability and reproducibility of the PD-L1 expression data on tumor cells with and without immune cells.

Clinically, ORR, PFS and OS (although not statistically significant) were improved in the subset of patients with CPS PD-L1+ compared with the PD-L1 population likely to benefit from treatment with pembrolizumab. Moreover, another important point is the possibility that CPS may be applied to cytologic specimens since it is independent of the tissue structure, offering the promise of a future advantage for cytologic or tissue tumor samples.

As substitutes for these approved assays, we have recently witnessed a commercial proliferation of low-cost LDTs, even after a rigorous validation of the proposed antibodies [71]. Adam et al. [72] compared five PD-L1 antibodies (28-8, 22C3, E1L3N, SP142 and SP263) on three IHC platforms for evaluating the PD-L1 percentage. The results achieved in this study showed that there is a high concordance between all antibodies and used platforms, though $50 \%$ of LDTs did not have adequate comparative technical performance [72].

Similar results have been reported in the harmonization study performed by Scheel et al. [73], where the PD-L1 level was evaluated using 4 assays (28-8, 22C3, SP142 and SP263) and 11 LDTs. For PD-L1 levels $\geq 1 \%$ and $\geq 50 \%$, good concordance between the different assays has been reported, with moderate concordance between LDTs and 22C3 assays. These 11 LDTs showed different results: 6 showed tumor cell staining like the 28-8 and 22C3 assays, while 5 LDTs detected weak PD-L1 staining compared with the assays validated by the FDA [73]. The literature demonstrated comparable performance regarding the used antibodies and platform. However, the comparison is affected by 
the variable definitions of positivity for each companion or complementary diagnostic and also there is not a clear gold standard comparator, except the commercial kits. In the USA, the College of American Pathologists (CAP) has drafted the requirements for the validations of IHC LDTs. These recommendations may be difficult to fulfill for single laboratories for several reasons. Some of these are represented by the many analyses for the biomarker, in both positive and negative samples, or the variable definition of positive and negative staining [74].

Other large organizations, such as the North Quality Control or UK National External Quality Assessment Service (UKNEQAS), independently agreed that the laboratories using LDTs had a serious possibility of failing compared with laboratories that use commercial kits $[75,76]$.

Thus, a possibility for the standardization of PD-L1 LDTs is actually the comparison with the commercial assay, such as PD-L1 IHC22C3 pharmDx and TPSs, to detect any LDT misclassification. A standardization of the platform and staining conditions is desirable for a reproducible result, which will be performed by international proficiency testing to promote high quality and equivalence among PD-L1 IHC assays.

\section{SUMMARY AND RECOMMENDATIONS}

The introduction of immune checkpoint inhibitors has recently become a promising frontier for the treatment of NCSLC. Since pembrolizumab inhibits PD1/PD-L1 interactions, PD-L1 IHC was developed as a biomarker to help discern responder from non-responder patients. Evaluation of PD-L1 expression by using IHC is currently the only biomarker approved for clinical use and therefore plays a central role in treatment decision-making for patients with advanced NSCLC.

However, although PD-L1 expression demonstrated a predictive value, its ability to accurately predict the response to immune checkpoint inhibitors can be suboptimal because of several limitations inherent to the tissue sampling, IHC detection methods and antibodies used in addition to dynamic and heterogeneous PD-L1 expression during cancer evolution and treatment in individual patients.

Therefore, in the future, the identification of more precise and dynamic predictive biomarkers for checkpoint blockades is an important goal to identify which populations are likely to experience a response to immunotherapy and to maximize the therapeutic benefits. Meanwhile, PD-L1 expression should be evaluated using a validated companion diagnostic to optimally predict an appropriate patient selection for PD-1/PD-L1 blockade and to select more sensitive patients.

\section{ACKNOWLEDGEMENTS}

Funding. This work was supported by the Consorzio Interuniversitario Nazionale per la Bio-Oncologia (CINBO). No Rapid Service Fee or Open Access fee was received by the journal for the publication of this article.

Additional Assistance. The authors thank Dr. Chiara Drago for the English language revision.

Authorship. All named authors meet the International Committee of Medical Journal Editors (ICMJE) criteria for authorship for this article, take responsibility for the integrity of the work as a whole and have given their approval for this version to be published.

Disclosures. All named authors Lorena Incorvaia, Daniele Fanale, Giuseppe Badalamenti, Nadia Barraco, Marco Bono, Lidia Rita Corsini, Antonio Galvano, Valerio Gristina, Angela Listì, Salvatore Vieni, Stefania Gori, Viviana Bazan and Antonio Russo have nothing to disclose.

Compliance with Ethics Guidelines. This article is based on previously conducted studies and does not contain any studies with human participants or animals performed by any of the authors. 
Peer Review. During the peer review process, the manufacturer of the agent under review was offered an opportunity to comment on the article. Changes resulting from comments received were made by the authors based on their scientific and editorial merit.

Open Access. This article is distributed under the terms of the Creative Commons Attribution-NonCommercial 4.0 International License (http://creativecommons.org/licenses/ by-nc/4.0/), which permits any noncommercial use, distribution, and reproduction in any medium, provided you give appropriate credit to the original author(s) and the source, provide a link to the Creative Commons license, and indicate if changes were made.

\section{REFERENCES}

1. Smyth MJ, Ngiow SF, Ribas A, Teng MWL. Combination cancer immunotherapies tailored to the tumour microenvironment. Nat Rev Clin Oncol. 2015;13(3):143-58. https://doi.org/10.1038/nrclino nc.2015.209.

2. Van der Jeught $\mathrm{K}$, Bialkowski L, Daszkiewicz L, Broos K, Goyvaerts C, Renmans D, et al. Targeting the tumor microenvironment to enhance antitumor immune responses. Oncotarget. 2015. https:// doi.org/10.18632/oncotarget.3204.

3. Melero I, Berman DM, Aznar MA, Korman AJ, Gracia JLP, Haanen J. Evolving synergistic combinations of targeted immunotherapies to combat cancer. Nat Rev Cancer. 2015;15(8):457-72. https:// doi.org/10.1038/nrc3973.

4. Zavala VA, Kalergis AM. New clinical advances in immunotherapy for the treatment of solid tumours. Immunology. 2015;145(2):182-201. https://doi. org/10.1111/imm.12459.

5. He J, Hu Y, Hu M, Li B. Development of PD-1/PD-L1 pathway in tumor immune microenvironment and treatment for non-small cell lung cancer. Sci Rep. 2015. https://doi.org/10.1038/srep13110.

6. Guo L, Zhang H, Chen B. Nivolumab as programmed death-1 (PD-1) inhibitor for targeted immunotherapy in tumor. J Cancer. 2017;8(3):410-6. https://doi. org/10.7150/jca.17144.

7. Incorvaia L, Bronte G, Bazan V, Badalamenti G, Rizzo S, Pantuso G, et al. Beyond evidence-based data: scientific rationale and tumor behavior to drive sequential and personalized therapeutic strategies for the treatment of metastatic renal cell carcinoma. Oncotarget. 2016. https://doi.org/10. 18632/oncotarget.7267.

8. Camisaschi C, Vallacchi V, Castelli C, Rivoltini L, Rodolfo M. Immune cells in the melanoma microenvironment hold information for prediction of the risk of recurrence and response to treatment. Expert Rev Mol Diagn. 2014;14(6):643-6. https:// doi.org/10.1586/14737159.2014.928206.

9. Chen L, Flies DB. Molecular mechanisms of T cell co-stimulation and co-inhibition. Nat Rev Immunol. 2013;13(4):227-42. https://doi.org/10.1038/ nri3405.

10. Curtsinger JM, Mescher MF. Inflammatory cytokines as a third signal for T cell activation. Curr Opin Immunol. 2010;22(3):333-40. https://doi.org/10. 1016/j.coi.2010.02.013.

11. Zitvogel L, Kroemer G. Targeting PD-1/PD-L1 interactions for cancer immunotherapy. OncoImmunology. 2014;1(8):1223-5. https://doi.org/10. 4161/onci.21335.

12. Badalamenti G, Fanale D, Incorvaia L, Barraco N, Listì A, Maragliano R, et al. Role of tumor-infiltrating lymphocytes in patients with solid tumors: can a drop dig a stone? Cell Immunol. 2018. https://doi. org/10.1016/j.cellimm.2018.01.013.

13. Beatty GL, Gladney WL. Immune escape mechanisms as a guide for cancer immunotherapy. Clin Cancer Res. 2014;21(4):687-92. https://doi.org/10. 1158/1078-0432.ccr-14-1860.

14. Chen L, Han X. Anti-PD-1/PD-L1 therapy of human cancer: past, present, and future. J Clin Investig. 2015;125(9):3384-91. https://doi.org/10.1172/jci8 0011.

15. Riley JL. PD-1 signaling in primary T cells. Immunol Rev. 2009;229(1):114-25. https://doi.org/10.1111/j. 1600-065X.2009.00767.x.

16. Parry RV, Chemnitz JM, Frauwirth KA, Lanfranco AR, Braunstein I, Kobayashi SV, et al. CTLA-4 and PD-1 receptors inhibit T-cell activation by distinct mechanisms. Mol Cell Biol. 2005;25(21):9543-53. https:// doi.org/10.1128/mcb.25.21.9543-9553.2005.

17. Robert C, Long GV, Brady B, Dutriaux C, Maio M, Mortier L, et al. Nivolumab in previously untreated melanoma without BRAF mutation. N Engl J Med. 2015;372(4):320-30. https://doi.org/10.1056/NEJM oa1412082.

18. Weber JS, D'Angelo SP, Minor D, Hodi FS, Gutzmer R, Neyns B, et al. Nivolumab versus chemotherapy 
in patients with advanced melanoma who progressed after anti-CTLA-4 treatment (CheckMate 037): a randomised, controlled, open-label, phase 3 trial. Lancet Oncol. 2015;16(4):375-84. https://doi. org/10.1016/s1470-2045(15)70076-8.

19. Guo Y, Wang AY. Novel immune check-point regulators in tolerance maintenance. Front Immunol. 2015. https://doi.org/10.3389/fimmu.2015.00421.

20. Peggs KS, Quezada SA, Korman AJ, Allison JP. Principles and use of anti-CTLA4 antibody in human cancer immunotherapy. Curr Opin Immunol. 2006;18(2):206-13. https://doi.org/10.1016/j. coi.2006.01.011.

21. Abiko K, Matsumura N, Hamanishi J, Horikawa N, Murakami R, Yamaguchi K, et al. IFN- $\gamma$ from lymphocytes induces PD-L1 expression and promotes progression of ovarian cancer. $\mathrm{Br} \mathrm{J}$ Cancer. 2015;112(9):1501-9. https://doi.org/10.1038/bjc. 2015.101.

22. Ayers M, Lunceford J, Nebozhyn M, Murphy E, Loboda A, Kaufman DR, et al. IFN- $\gamma$-related mRNA profile predicts clinical response to PD-1 blockade. J Clin Investig. 2017;127(8):2930-40. https://doi. org/10.1172/jci91190.

23. Patel SP, Kurzrock R. PD-L1 expression as a predictive biomarker in cancer immunotherapy. Mol Cancer Ther. 2015;14(4):847-56. https://doi.org/ 10.1158/1535-7163.mct-14-0983.

24. Reck M, Rodríguez-Abreu D, Robinson AG, Hui R, Csőszi T, Fülöp A, et al. Pembrolizumab versus chemotherapy for PD-L1-positive non-small-cell lung cancer. N Engl J Med. 2016;375(19):1823-33. https://doi.org/10.1056/NEJMoa1606774.

25. Corsini LR, Fanale D, Passiglia F, Incorvaia L, Gennusa $\mathrm{V}$, Bazan $\mathrm{V}$, et al. Monoclonal antibodies for the treatment of non-hematological tumors: a safety review. Expert Opin Drug Saf. 2018;17(12):1197-209. https://doi.org/10.1080/ 14740338.2018.1550068.

26. Passiglia F, Galvano A, Rizzo S, Incorvaia L, Listì A, Bazan $\mathrm{V}$, et al. Looking for the best immunecheckpoint inhibitor in pre-treated NSCLC patients: an indirect comparison between nivolumab, pembrolizumab and atezolizumab. Int $\mathrm{J}$ Cancer. 2018;142(6):1277-84. https://doi.org/10.1002/ijc. 31136.

27. Wolchok JD, Chiarion-Sileni V, Gonzalez R, Rutkowski P, Grob J-J, Cowey CL, et al. Overall survival with combined nivolumab and ipilimumab in advanced melanoma. N Engl J Med. 2017; 377(14):1345-56. https://doi.org/10.1056/NEJMoa1 709684 .
28. Larkin J, Chiarion-Sileni V, Gonzalez R, Grob JJ, Cowey CL, Lao CD, et al. Combined nivolumab and ipilimumab or monotherapy in untreated melanoma. N Engl J Med. 2015;373(1):23-34. https:// doi.org/10.1056/NEJMoa1504030.

29. Cavanna L, Citterio C, Orlandi E. Immune checkpoint inhibitors in EGFR-mutation positive TKItreated patients with advanced non-small-cell lung cancer network meta-analysis. Oncotarget. 2018. https://doi.org/10.18632/oncotarget.26541.

30. Robert C, Long GV, Schachter J, Arance A, Grob JJ, Mortier L, et al. Long-term outcomes in patients (pts) with ipilimumab (ipi)-naive advanced melanoma in the phase 3 KEYNOTE-006 study who completed pembrolizumab (pembro) treatment. J Clin Oncol. 2017;35(15_suppl):9504. https://doi. org/10.1200/jco.2017.35.15_suppl.9504.

31. Bian B, Fanale D, Dusetti N, Roque J, Pastor S, Chretien A-S, et al. Prognostic significance of circulating PD-1, PD-L1, pan-BTN3As, BTN3A1 and BTLA in patients with pancreatic adenocarcinoma. OncoImmunology. 2019;8(4):e1561120. https:// doi.org/10.1080/2162402x.2018.1561120.

32. Teixidó C, Vilariño N, Reyes R, Reguart N. PD-L1 expression testing in non-small cell lung cancer. Ther Adv Med Oncol. 2018;10:175883591876349. https://doi.org/10.1177/1758835918763493.

33. Scheel AH, Schäfer SC. Current PD-L1 immunohistochemistry for non-small cell lung cancer. J Thorac Dis. 2018;10(3):1217-9. https://doi.org/10.21037/ jtd.2018.02.38.

34. Sui H, Ma N, Wang Y, Li H, Liu X, Su Y, et al. AntiPD-1/PD-L1 therapy for non-small-cell lung cancer: toward personalized medicine and combination strategies. J Immunol Res. 2018;2018:1-17. https:// doi.org/10.1155/2018/6984948.

35. Roach C, Zhang N, Corigliano E, Jansson M, Toland $G$, Ponto G, et al. Development of a companion diagnostic PD-L1 immunohistochemistry assay for pembrolizumab therapy in non-small-cell lung cancer. Appl Immunohistochem Mol Morphol. 2016;24(6):392-7. https://doi.org/10.1097/pai. 0000000000000408 .

36. Thunnissen E, de Langen AJ, Smit EF. PD-L1 IHC in NSCLC with a global and methodological perspective. Lung Cancer. 2017;113:102-5. https://doi.org/ 10.1016/j.lungcan.2017.09.010.

37. Garon EB, Rizvi NA, Hui R, Leighl N, Balmanoukian AS, Eder JP, et al. Pembrolizumab for the treatment of non-small-cell lung cancer. $\mathrm{N}$ Engl J Med. 2015;372(21):2018-28. https://doi.org/10.1056/ NEJMoa1501824. 
38. Herbst RS, Baas P, Kim D-W, Felip E, Pérez-Gracia JL, Han J-Y, et al. Pembrolizumab versus docetaxel for previously treated, PD-L1-positive, advanced nonsmall-cell lung cancer (KEYNOTE-010): a randomised controlled trial. Lancet. 2016;387(10027):1540-50. https://doi.org/10.1016/ s0140-6736(15)01281-7.

39. Herbst RS, Baas P, Perez-Gracia JL, Felip E, Kim DW, Han JY, et al. Use of archival versus newly collected tumor samples for assessing PD-L1 expression and overall survival: an updated analysis of KEYNOTE010 trial. Ann Oncol. 2019;30(2):281-9. https://doi. org/10.1093/annonc/mdy545.

40. Russo A, Franchina T, Ricciardi GRR, Toscano G, Schifano S, Lo Certo G, et al. The changing scenario of 1st line therapy in non-oncogene addicted NSCLCs in the era of immunotherapy. Crit Rev Oncol Hematol. 2018;130:1-12. https://doi.org/10. 1016/j.critrevonc.2018.06.007.

41. Reck M, Rodríguez-Abreu D, Robinson AG, Hui R, Csőszi T, Fülöp A, et al. Updated Analysis of KEYNOTE-024: pembrolizumab versus platinum-based chemotherapy for advanced non-small-cell lung cancer with PD-L1 Tumor Proportion Score of 50\% or greater. J Clin Oncol. 2019;37(7):537-46. https:// doi.org/10.1200/jco.18.00149.

42. Mok TSK, Wu Y-L, Kudaba I, Kowalski DM, Cho BC, Turna HZ, et al. Pembrolizumab versus chemotherapy for previously untreated, PD-L1-expressing, locally advanced or metastatic non-smallcell lung cancer (KEYNOTE-042): a randomised, open-label, controlled, phase 3 trial. The Lancet. 2019;393(10183):1819-30. https://doi.org/10.1016/ s0140-6736(18)32409-7.

43. Karim S, Leighl N. Pembrolizumab for the treatment of thoracic malignancies: current landscape and future directions. Future Oncol. 2016;12(1):9-23. https://doi.org/10.2217/fon.15. 294.

44. Sorensen SF, Zhou W, Dolled-Filhart M, Georgsen JB, Wang Z, Emancipator K, et al. PD-L1 expression and survival among patients with advanced nonsmall cell lung cancer treated with chemotherapy. Transl Oncol. 2016;9(1):64-9. https://doi.org/10. 1016/j.tranon.2016.01.003.

45. Buchbinder EI, Desai A. CTLA-4 and PD-1 pathways. Am J Clin Oncol. 2016;39(1):98-106. https:// doi.org/10.1097/coc.0000000000000239.

46. Ilie M, Hofman V, Dietel M, Soria J-C, Hofman P. Assessment of the PD-L1 status by immunohistochemistry: challenges and perspectives for therapeutic strategies in lung cancer patients. Virchows Arch. 2016;468(5):511-25. https://doi.org/10.1007/ s00428-016-1910-4.
47. Borghaei H, Langer CJ, Gadgeel S, Papadimitrakopoulou VA, Patnaik A, Powell SF, et al. 24-Month overall survival from KEYNOTE-021 Cohort G: pemetrexed and carboplatin with or without pembrolizumab as first-line therapy for advanced nonsquamous non-small cell lung cancer. J Thorac Oncol. 2019;14(1):124-9. https://doi.org/ 10.1016/j.jtho.2018.08.004.

48. Gandhi L, Rodríguez-Abreu D, Gadgeel S, Esteban E, Felip E, De Angelis F, et al. Pembrolizumab plus chemotherapy in metastatic non-small-cell lung cancer. N Engl J Med. 2018;378(22):2078-92. https://doi.org/10.1056/NEJMoa1801005.

49. Paz-Ares L, Luft A, Vicente D, Tafreshi A, Gümüş M, Mazières $\mathrm{J}$, et al. Pembrolizumab plus chemotherapy for squamous non-small-cell lung cancer. N Engl J Med. 2018;379(21):2040-51. https://doi. org/10.1056/NEJMoa1810865.

50. Zhang Y, Wang L, Li Y, Pan Y, Hu H, Li H, et al. Protein expression of programmed death 1 ligand 1 and ligand 2 independently predict poor prognosis in surgically resected lung adenocarcinoma. OncoTargets Ther. 2014. https://doi.org/10.2147/ ott.s59959.

51. Ilie M, Long-Mira E, Bence C, Butori C, Lassalle S, Bouhlel L, et al. Comparative study of the PD-L1 status between surgically resected specimens and matched biopsies of NSCLC patients reveal major discordances: a potential issue for anti-PD-L1 therapeutic strategies. Ann Oncol. 2016;27(1):147-53. https://doi.org/10.1093/annonc/mdv489.

52. Dolled-Filhart M, Locke D, Murphy T, Lynch F, Yearley JH, Frisman D, et al. Development of a prototype immunohistochemistry assay to measure programmed death ligand-1 expression in tumor tissue. Arch Pathol Lab Med. 2016;140(11):1259-66. https://doi.org/10.5858/ arpa.2015-0544-OA.

53. Mino-Kenudson M, Mino-Kenudson M. Programmed cell death ligand-1 (PD-L1) expression by immunohistochemistry: could it be predictive and/ or prognostic in non-small cell lung cancer? Cancer Biol Med. 2016;13(2):157-70. https://doi.org/10. 20892/j.issn.2095-3941.2016.0009.

54. Büttner R, Gosney JR, Skov BG, Adam J, Motoi N, Bloom KJ, et al. Programmed death-ligand 1 immunohistochemistry testing: a review of analytical assays and clinical implementation in nonsmall-cell lung cancer. J Clin Oncol. 2017;35(34):3867-76. https://doi.org/10.1200/jco. 2017.74.7642.

55. Dolled-Filhart M, Roach C, Toland G, Stanforth D, Jansson M, Lubiniecki GM, et al. Development of a companion diagnostic for pembrolizumab in non- 
small cell lung cancer using immunohistochemistry for programmed death ligand-1. Arch Pathol Lab Med. 2016;140(11):1243-9. https://doi.org/10. 5858/arpa.2015-0542-OA.

56. Novotny JF, Cogswell J, Inzunza H, Harbison C, Horak C, Averbuch S. Establishing a complementary diagnostic for anti-PD-1 immune checkpoint inhibitor therapy. Ann Oncol. 2016;27(10):1966-9. https://doi.org/10.1093/annonc/mdw288.

57. Hirsch FR, McElhinny A, Stanforth D, RangerMoore J, Jansson M, Kulangara K, et al. PD-L1 immunohistochemistry assays for lung cancer: results from phase 1 of the blueprint PD-L1 IHC assay comparison project. J Thorac Oncol. 2017;12(2):208-22. https://doi.org/10.1016/j.jtho. 2016.11.2228.

58. Tsao MS, Kerr KM, Kockx M, Beasley M-B, Borczuk AC, Botling J, et al. PD-L1 immunohistochemistry comparability study in real-life clinical samples: results of blueprint phase 2 project. J Thorac Oncol. 2018;13(9):1302-11. https://doi.org/10.1016/j.jtho. 2018.05.013.

59. Li C, Huang C, Mok TS, Zhuang W, Xu H, Miao Q, et al. Comparison of 22C3 PD-L1 expression between surgically resected specimens and paired tissue microarrays in non-small cell lung cancer. J Thorac Oncol. 2017;12(10):1536-43. https://doi. org/10.1016/j.jtho.2017.07.015.

60. Skov BG, Skov T. Paired comparison of PD-L1 expression on cytologic and histologic specimens from malignancies in the lung assessed with PD-L1 IHC 28-8pharmDx and PD-L1 IHC 22C3pharmDx. Appl Immunohistochem Mol Morphol. 2017;25(7):453-9. https://doi.org/10.1097/pai. 0000000000000540 .

61. Heymann JJ, Bulman WA, Swinarski D, Pagan CA, Crapanzano JP, Haghighi M, et al. PD-L1 expression in non-small cell lung carcinoma: comparison among cytology, small biopsy, and surgical resection specimens. Cancer Cytopathol. 2017;125(12):896-907. https://doi.org/10.1002/ cncy.21937.

62. Wang H, Spatz A. Making cytology specimens solid materials for testing predictive marker of immunotherapy in NSCLC. Oncotarget. 2018. https://doi.org/10.18632/oncotarget.26261.

63. Midha A, Sharpe A, Scott M, Walker J, Shi K, Ballas $M$, et al. PD-L1 expression in advanced NSCLC: primary lesions versus metastatic sites and impact of sample age. J Clin Oncol. 2016;34(15_suppl):3025. https://doi.org/10.1200/jco.2016.34. 15_suppl.3025.
64. Kerr KM, Hirsch FR. Programmed death ligand-1 immunohistochemistry: friend or foe? Arch Pathol Lab Med. 2016;140(4):326-31. https://doi.org/10. 5858/arpa.2015-0522-SA.

65. Cree IA, Booton R, Cane P, Gosney J, Ibrahim M, Kerr K, et al. PD-L1 testing for lung cancer in the UK: recognizing the challenges for implementation. Histopathology. 2016;69(2):177-86. https://doi. org/10.1111/his.12996.

66. Sharpe AH, Wherry EJ, Ahmed R, Freeman GJ. The function of programmed cell death 1 and its ligands in regulating autoimmunity and infection. Nat Immunol. 2007;8(3):239-45. https://doi.org/10. 1038/ni1443.

67. Yu H, Boyle TA, Zhou C, Rimm DL, Hirsch FR. PDL1 expression in lung cancer. J Thorac Oncol. 2016;11(7):964-75. https://doi.org/10.1016/j.jtho. 2016.04.014.

68. Garon EB. Current perspectives in immunotherapy for non-small cell lung cancer. Semin Oncol. 2015;42:S11-8. https://doi.org/10.1053/j. seminoncol.2015.09.019.

69. Hui R, Garon EB, Goldman JW, Leighl NB, Hellmann MD, Patnaik A, et al. Pembrolizumab as firstline therapy for patients with PD-L1-positive advanced non-small cell lung cancer: a phase 1 trial. Ann Oncol. 2017;28(4):874-81. https://doi. org/10.1093/annonc/mdx008.

70. Kulangara K, Zhang N, Corigliano E, Guerrero L, Waldroup S, Jaiswal D, et al. Clinical utility of the combined positive score for programmed death ligand-1 expression and the approval of pembrolizumab for treatment of gastric cancer. Arch Pathol Lab Med. 2019;143(3):330-7. https://doi. org/10.5858/arpa.2018-0043-OA.

71. Hirsch FR, Suda K, Wiens J, Bunn PA. New and emerging targeted treatments in advanced nonsmall-cell lung cancer. Lancet. 2016;388(10048):1012-24. https://doi.org/10.1016/ s0140-6736(16)31473-8.

72. Adam J, Le Stang N, Rouquette I, Cazes A, Badoual $\mathrm{C}$, Pinot-Roussel $\mathrm{H}$, et al. Multicenter harmonization study for PD-L1 IHC testing in non-small-cell lung cancer. Ann Oncol. 2018;29(4):953-8. https:// doi.org/10.1093/annonc/mdy014.

73. Scheel AH, Dietel M, Heukamp LC, Jöhrens K, Kirchner $\mathrm{T}$, Reu S, et al. Harmonized PD-L1 immunohistochemistry for pulmonary squamouscell and adenocarcinomas. Mod Pathol. 2016;29(10):1165-72. https://doi.org/10.1038/mod pathol.2016.117. 
74. Lin F, Chen Z. Standardization of diagnostic immunohistochemistry: literature review and geisinger experience. Arch Pathol Lab Med. 2014;138(12):1564-77. https://doi.org/10.5858/ar pa.2014-0074-RA.

75. Vyberg $M$, Nielsen S. Proficiency testing in immunohistochemistry-experiences from nordic immunohistochemical quality control (NordiQC).
Virchows Arch. 2015;468(1):19-29. https://doi.org/ 10.1007/s00428-015-1829-1.

76. Ibrahim M, Parry S, Wilkinson D, Bilbe N, Allen D, Forrest $\mathrm{S}$, et al. ALK immunohistochemistry in NSCLC: discordant staining can impact patient treatment regimen. J Thorac Oncol. 2016;11(12):2241-7. https://doi.org/10.1016/j.jtho. 2016.07.012. 